\title{
Comparative evaluation of three clinical decision support systems: prospective screening for medication errors in 100 medical inpatients
}

\author{
Fritz, Daniela ; Ceschi, Alessandro ; Curkovic, Ivanka ; Huber, Martin ; Egbring, Marco ; \\ Kullak-Ublick, Gerd A ; Russmann, Stefan
}

\begin{abstract}
PURPOSE: Clinical decision support systems (CDSS) are promoted as powerful screening tools to improve pharmacotherapy. The aim of our study was to evaluate the potential contribution of CDSS to patient management in clinical practice. METHODS: We prospectively analyzed the pharmacotherapy of 100 medical inpatients through the parallel use of three CDSS, namely, Pharmavista, DrugReax, and TheraOpt. After expert discussion that also considered all patient-specific clinical information, we selected apparently relevant alerts, issued suitable recommendations to physicians, and recorded subsequent prescription changes. RESULTS: For 100 patients with a median of eight concomitant drugs, Pharmavista, DrugReax, and TheraOpt generated a total of 53, 362, and 328 interaction alerts, respectively. Among those we identified and forwarded 33 clinically relevant alerts to the attending physician, resulting in 19 prescription changes. Four adverse drug events were associated with interactions. The proportion of clinically relevant alerts among all alerts (positive predictive value) was 5.7, 8.0, and 7.6\%, and the sensitivity to detect all 33 relevant alerts was 9.1, 87.9, and 75.8\% for Pharmavista, DrugReax and TheraOpt, respectively. TheraOpt recommended 31 dose adjustments, of which we considered 11 to be relevant; three of these were followed by dose reductions. CONCLUSIONS: CDSS are valuable screening tools for medication errors, but only a small fraction of their alerts appear relevant in individual patients. In order to avoid overalerting CDSS should use patient-specific information and management-oriented classifications. Comprehensive information should be displayed on-demand, whereas a limited number of computer-triggered alerts that have management implications in the majority of affected patients should be based on locally customized and supported algorithms.
\end{abstract}

DOI: https://doi.org/10.1007/s00228-012-1241-6

Posted at the Zurich Open Repository and Archive, University of Zurich

ZORA URL: https://doi.org/10.5167/uzh-63199

Journal Article

Published Version

Originally published at:

Fritz, Daniela; Ceschi, Alessandro; Curkovic, Ivanka; Huber, Martin; Egbring, Marco; Kullak-Ublick, Gerd A; Russmann, Stefan (2012). Comparative evaluation of three clinical decision support systems: prospective screening for medication errors in 100 medical inpatients. European Journal of Clinical Pharmacology, 68(8):1209-1219.

DOI: https://doi.org/10.1007/s00228-012-1241-6 


\title{
Comparative evaluation of three clinical decision support systems: prospective screening for medication errors in $\mathbf{1 0 0}$ medical inpatients
}

\author{
Daniela Fritz • Alessandro Ceschi • Ivanka Curkovic • \\ Martin Huber • Marco Egbring • \\ Gerd A. Kullak-Ublick • Stefan Russmann
}

Received: 27 October 2011 / Accepted: 1 February 2012 / Published online: 29 February 2012

(C) Springer-Verlag 2012

\begin{abstract}
Purpose Clinical decision support systems (CDSS) are promoted as powerful screening tools to improve pharmacotherapy. The aim of our study was to evaluate the potential contribution of CDSS to patient management in clinical practice.

Methods We prospectively analyzed the pharmacotherapy of 100 medical inpatients through the parallel use of three CDSS, namely, Pharmavista, DrugReax, and TheraOpt. After expert discussion that also considered all patient-specific clinical information, we selected apparently relevant alerts, issued suitable recommendations to physicians, and recorded subsequent prescription changes.

Results For 100 patients with a median of eight concomitant drugs, Pharmavista, DrugReax, and TheraOpt generated a total of 53, 362, and 328 interaction alerts, respectively. Among those we identified and forwarded 33 clinically relevant alerts to the attending physician, resulting in 19 prescription changes. Four adverse drug events were associated with interactions. The proportion of clinically relevant alerts among all alerts (positive predictive value) was 5.7, 8.0,
\end{abstract}

D. Fritz · A. Ceschi - I. Curkovic · M. Huber · M. Egbring ·

G. A. Kullak-Ublick $\cdot$ S. Russmann $(\bowtie)$

Department of Clinical Pharmacology and Toxicology,

University Hospital Zurich,

Rämistrasse 100,

8091 Zurich, Switzerland

e-mail: stefan.russmann@usz.ch

\section{A. Ceschi}

Swiss Toxicological Information Center,

Zurich, Switzerland

G. A. Kullak-Ublick $\cdot$ S. Russmann

Zurich Center for Integrative Human Physiology (ZIHP),

Zurich, Switzerland and $7.6 \%$, and the sensitivity to detect all 33 relevant alerts was $9.1,87.9$, and $75.8 \%$ for Pharmavista, DrugReax and TheraOpt, respectively. TheraOpt recommended 31 dose adjustments, of which we considered 11 to be relevant; three of these were followed by dose reductions.

Conclusions CDSS are valuable screening tools for medication errors, but only a small fraction of their alerts appear relevant in individual patients. In order to avoid overalerting CDSS should use patient-specific information and management-oriented classifications. Comprehensive information should be displayed on-demand, whereas a limited number of computer-triggered alerts that have management implications in the majority of affected patients should be based on locally customized and supported algorithms.

Keywords Clinical decision support software - Dose adjustment $\cdot$ Drug interactions

\section{Introduction}

Adverse drug events (ADE), which are an important cause of morbidity and mortality and result in increased healthcare costs, are a challenging problem in clinical patient care [1-7]. Drug interactions and dosing errors leading to ADE are of special interest because they represent preventable medication errors that are suitable targets for highly efficient automated interventions through computerized physician order entry (CPOE) with clinical decision support systems (CDSS). The efficacy of CDSS to modify physicians' behavior in clinical practice and thereby to reduce medication errors and improve monitoring of pharmacotherapy has been well established, whereas their efficacy to reduce 
$\mathrm{ADE}$ and costs is less well documented and needs further investigation [8-15]. In addition, few studies have compared the performance of different CDSS, and these were usually not conducted under real-life conditions. The classification and grading of medication errors is a complex and challenging task, and previous studies have reported major disagreements in the assessment of drug interactions between different CDSS [16-18] and specialists [19]. Furthermore, general assessments may not apply well to specific patients where prescribing clinicians also use additional complex non-standardized clinical information for management decisions [20, 21]. In general, it appears that most CDSS have a high sensitivity to detect drug interactions at the cost of low specificity to discriminate those interactions that are clinically relevant. In combination with an insufficient consideration of patient-specific factors by CDSS, this leads to indiscriminate overriding of alerts by prescribing clinicians, which jeopardizes the efficacy of CDSS to improve medication safety in clinical practice [22].

For our routine clinical pharmacology "safety ward rounds", we use several CDSS as an initial screening tool to search for drug interactions and dosing errors in hospitalized patients. However, given the limitations mentioned above, after automated screening we also access the electronic medical records of the respective patients in order to evaluate the clinical relevance of potential medication errors initially identified by the CDSS. Only if we conclude that a potential medication error is clinically relevant in the patient's individual clinical context do we alert the responsible physician and discuss appropriate alternatives. Because there is a need for systematic evaluations of the performance of different CDSS in real-life clinical settings, we have analyzed our experience with the use of different CDSS in combination with clinical pharmacology expertise for the identification and prevention of medication errors. The results of this analysis are reported here.

\section{Methods}

\section{Study design}

We present a prospective naturalistic analysis that evaluated the performance of CDSS used as part of our surveillance of pharmacotherapy at two general internal medicine wards of a tertiary care university hospital. Our surveillance has the aim to optimize the efficacy and safety of pharmacotherapy. Because this study is a systematic analysis of our established routine clinical practice it was exempt from ethical approval. A summary of the procedures is presented in Fig. 1. The study includes 100 consecutive patients for an evaluation of all concomitantly prescribed drugs with a focus on potential drug interactions. There were no formal exclusion criteria. A formal power analysis was not applicable in this descriptive pilot analysis, and the decision to include 100 patients was based on pragmatic and somewhat arbitrary grounds. As our surveillance is performed at certain days of the week and patients may also had been transferred from other wards, the day of analysis in relation to hospital admission varied. All concomitantly prescribed drugs were simultaneously analyzed with three different CDSS, i.e., Micromedex DRUGREAX, Atheso TheraOpt (which in the meantime has been taken over by ID Berlin), and Pharmavista [23-25]. Of note, Pharmavista did not allow the entry of more than eight concomitantly used drugs at any one time, and for patients using more than eight drugs an interaction analysis could therefore not be performed with Pharmavista. Automatically generated alerts from all three CDSS were documented. Subsequent to receiving the automatically generated alerts, at least one junior and one senior clinical pharmacologist from our department discussed and evaluated the clinical relevance of the alerts in the individual clinical context of each patient. Alerts were defined as clinically relevant if we considered a change of therapy to be necessary. For that purpose we also accessed the hospital's clinical information system containing all electronic drug prescriptions, medical reports, and laboratory results. We then forwarded only those alerts that we presumed to be clinically relevant to the attending physician, usually by personal communication during ward rounds or over the phone, and made an entry into the electronic patient record. If appropriate, we also provided additional information and management recommendations, including possible alternative therapies and monitoring. Thereafter, we followed the electronic prescribing record for those patients until hospital discharge in order to document whether prescriptions had been changed in accordance with our recommendations. We also specifically searched medical reports and laboratory results for signs and symptoms of $\mathrm{ADE}$ that may have resulted from prescriptions addressed in the alerts.

\section{Outcomes and data analysis}

Primary outcomes of the study were the comparative number of alerts generated by each CDSS and the fractions thereof that we considered to be clinically relevant and therefore forwarded to the attending physicians. In order to compare the performance of the three CDSS we calculated their sensitivity and positive predictive value (PPV) in terms of their identification of clinically relevant drug interactions as follows. Sensitivity $=$ number of interactions among all 
Fig. 1 Procedures. Overview of the pharmacotherapy evaluation process. CDSS Clinical decision support system

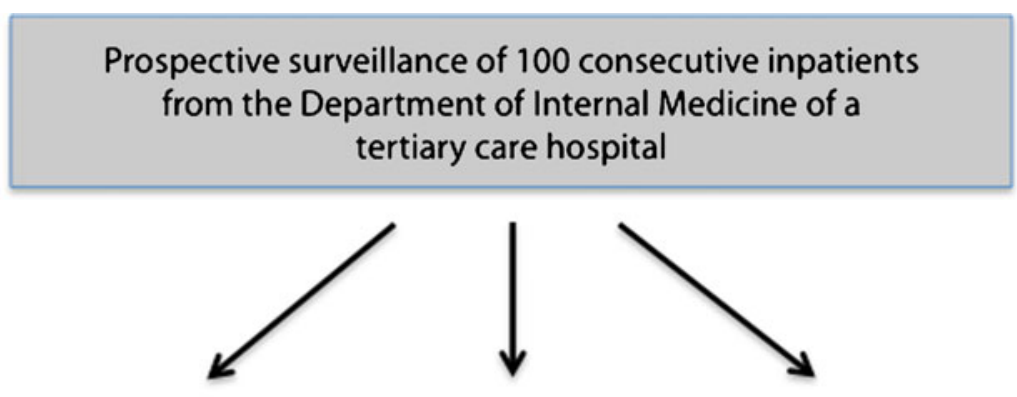

Simultaneous analysis of all current prescriptions with three different CDSS

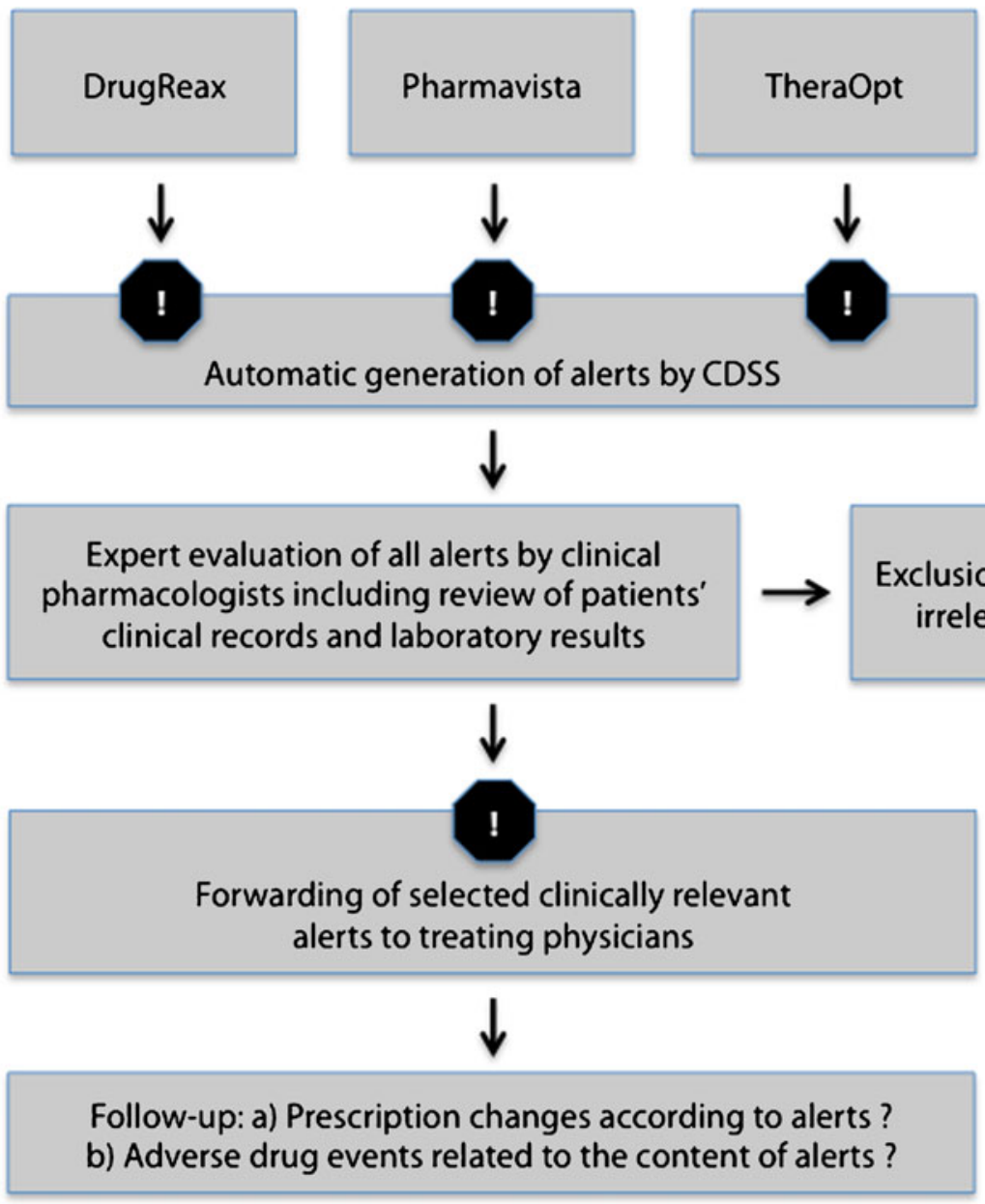

interactions evaluated as clinically relevant identified by the respective CDSS divided by all interactions evaluated as clinically relevant identified by any CDSS or during expert discussion; PPV = number of interactions identified by the respective CDSS and evaluated as clinically relevant divided by the number of interactions identified by the respective CDSS. We compared the sensitivity and PPV between different CDSS using the chi-square test.

Additional measures of interest included the number of actual medication changes in response to those alerts that we had forwarded to the attending physicians as well as stratified analyses, such as by CDSS grading, and the description of specific alerts and associated ADE. Data management, analyses, and construction of figures were done using STATA 11.2 for MacOS X (STATA Corp, College Station, TX).

\section{Results}

Patient characteristics and drug use

Demographic and clinical characteristics of all 100 included patients are presented in Table 1 . The median age was 
Table 1 Patient characteristics

\begin{tabular}{|c|c|}
\hline Characteristics & $n$ \\
\hline Total & 100 \\
\hline \multicolumn{2}{|l|}{ Sex } \\
\hline Female & 42 \\
\hline Male & 58 \\
\hline \multicolumn{2}{|l|}{ Age category (years) } \\
\hline$<50$ & 20 \\
\hline $50-59$ & 32 \\
\hline $60-69$ & 19 \\
\hline $70-79$ & 17 \\
\hline$\geq 80$ & 12 \\
\hline \multicolumn{2}{|c|}{ Time of pharmacotherapy evaluation (days after admission) } \\
\hline $0-1$ & 16 \\
\hline $2-4$ & 33 \\
\hline $5-9$ & 37 \\
\hline$\geq 10$ & 14 \\
\hline \multicolumn{2}{|l|}{ Disease categories $^{\mathrm{a}}$} \\
\hline Gastroenterology and hepatology & 25 \\
\hline Nephrology & 24 \\
\hline Internal medicine & 17 \\
\hline Cardiology & 11 \\
\hline Angiology & 6 \\
\hline Oncology & 4 \\
\hline Pneumology & 4 \\
\hline Endocrinology & 3 \\
\hline Hematology & 3 \\
\hline Infectiology & 2 \\
\hline Immunology & 1 \\
\hline \multicolumn{2}{|c|}{ Renal function $\left(\mathrm{GFR}, \mathrm{ml} / \mathrm{min} / 1.73 \mathrm{~m}^{2}\right)^{\mathrm{b}}$} \\
\hline$\geq 60$ & 56 \\
\hline $30-59$ & 22 \\
\hline$<30$, no dialysis & 11 \\
\hline Dialysis & 11 \\
\hline \multicolumn{2}{|l|}{ Liver disease with CHILD score $\geq 7$} \\
\hline Yes & 11 \\
\hline No & 89 \\
\hline \multicolumn{2}{|l|}{ Malignancy } \\
\hline Yes & 25 \\
\hline No & 75 \\
\hline \multicolumn{2}{|l|}{ Transplantation } \\
\hline Liver & 2 \\
\hline Lung & 5 \\
\hline Kidney & 16 \\
\hline
\end{tabular}

${ }^{\text {a }}$ Only one primary diagnosis per patient

${ }^{\mathrm{b}}$ Most recent glomerular filtration rate (GFR) before pharmacotherapy evaluation according to the MDRD-formula

59.3 years (range $23-86$ ), and in 70 patients the pharmacotherapy was analyzed between days 2 and 9 after admission (median and mean 5 and 6.5 days, respectively). Forty-four patients had impaired renal function with a glomerular filtration rate (GFR) of $<60 \mathrm{ml} / \mathrm{min}, 11$ had liver disease with a CHILD score of $\geq 7,23$ had undergone organ transplantation at any time in the past, and 25 patients had malignant tumors.

Table 2 presents the most commonly prescribed drugs in the study population. Overall, there were 892 prescriptions in 100 patients. Based on drug classification, antibiotics ranked as the most frequently prescribed drug class, accounting for 110 prescriptions $(12.3 \%)$ in 50 patients, followed by heparins, proton pump inhibitors, diuretics, and beta-blockers.

A histogram of the polypharmacy distribution is shown in Fig. 2. The mean and median number of concomitant substances prescribed to each patient was 8.9 and 8 , respectively.

\section{Drug interactions}

The correlation between polypharmacy and the mean number of identified interactions per patient by each CDSS is shown in Fig. 3. As expected, based on the exponential increase of possible combinations, increasing polypharmacy was also associated with a pronounced increase of identified interactions. Because Pharmavista can only analyze up to eight concomitantly prescribed drugs, there are no results from Pharmavista for patients receiving more than eight drugs. Consequently, the overall number of interactions identified by Pharmavista was much lower compared to DrugReax or Theraopt (Pharmavista 53, DrugReax 362, TheraOpt 328). As expected, for patients receiving up to eight drugs, differences in the number of identified interactions were smaller (Pharmavista 53, DrugReax 75, TheraOpt 56). An overview of the identification of drug interactions by each CDSS, including our subsequent expert evaluation and implementation of the resulting recommendations by the treating physicians, is provided in Table 3. Among all interaction alerts generated by any CDSS, we evaluated 33 interactions as clinically relevant in the individual clinical context of the respective patients. Of note, when a CDSS classified an alert as severe and we did not forward this alert to the treating physician, the reason for our decision was documented. For example, there were five "severe" pharmacokinetic interaction alerts with cyclosporine, but therapeutic drug monitoring indicated the appropriate dose adjustment with concentrations in the therapeutic range. Other examples include the combination of several drugs that increase the risk of bleeding when there was an evidence-based indication for this combination, or the combination of several potassium-sparing drugs when potassium concentrations were indeed normal and stable. The proportion of generated alerts that we considered to be clinically 
Table 2 Prescription frequencies for different drug categories/classes ${ }^{\mathrm{a}}$

\begin{tabular}{|c|c|c|c|}
\hline \multirow[t]{2}{*}{ Drug classes } & \multicolumn{2}{|c|}{$\begin{array}{l}\text { Number of } \\
\text { prescriptions }\end{array}$} & \multirow{2}{*}{$\begin{array}{l}\text { Number of patients } \\
\text { with prescriptions } \\
n(=\%)\end{array}$} \\
\hline & $n$ & $\%$ & \\
\hline Total & 892 & 100 & 100 \\
\hline Antibiotics & 110 & 12.3 & 50 \\
\hline Heparins & 63 & 7.1 & 63 \\
\hline Proton pump inhibitors & 60 & 6.7 & 60 \\
\hline Diuretics & 52 & 5.8 & 40 \\
\hline Beta blockers & 43 & 4.8 & 43 \\
\hline Immunosuppressants & 40 & 4.5 & 23 \\
\hline HMG-CoA inhibitors & 38 & 4.3 & 38 \\
\hline Antiplatelets & 37 & 4.2 & 28 \\
\hline Corticosteroids systemic & 34 & 3.8 & 34 \\
\hline ACE inhibitors & 28 & 3.1 & 28 \\
\hline Vitamins & 28 & 3.1 & 28 \\
\hline Antivirals & 26 & 2.9 & 15 \\
\hline Calcium salts & 22 & 2.5 & 22 \\
\hline Laxatives & 22 & 2.5 & 18 \\
\hline Hormones & 20 & 2.2 & 17 \\
\hline Calcium channel blockers & 19 & 2.1 & 19 \\
\hline Antifungals & 16 & 1.8 & 12 \\
\hline Potassium salts & 16 & 1.8 & 16 \\
\hline Angiotensin renin blockers & 14 & 1.6 & 14 \\
\hline $\begin{array}{l}\text { Benzodiazepines and GABA } \\
\text { agonists }\end{array}$ & 14 & 1.6 & 13 \\
\hline Oral anticoagulants & 14 & 1.6 & 14 \\
\hline Insulins & 13 & 1.5 & 9 \\
\hline Analgetics metamizole & 11 & 1.2 & 11 \\
\hline Analgetics opioids & 10 & 1.1 & 10 \\
\hline Analgetics paracetamol & 9 & 1.0 & 9 \\
\hline Antidepressants & 9 & 1.0 & 7 \\
\hline Antiemetics & 9 & 1.0 & 8 \\
\hline Antiepileptics & 9 & 1.0 & 7 \\
\hline Oral glucose lowering agents & 9 & 1.0 & 7 \\
\hline Antiarrhythmics & 8 & 0.9 & 7 \\
\hline Antiasthmatics & 7 & 0.8 & 3 \\
\hline Magnesium salts & 7 & 0.8 & 7 \\
\hline Neuroleptics & 7 & 0.8 & 7 \\
\hline Antiparkinson drugs & 4 & 0.5 & 2 \\
\hline Uricostatics & 4 & 0.5 & 4 \\
\hline NSAIDs & 2 & 0.2 & 2 \\
\hline Other & 58 & 6.5 & 44 \\
\hline
\end{tabular}

HMG-Co, 3-Hydroxy-3-methylglutaryl-coenzyme A; ACE, angiotensin converting enzyme; GABA, gamma-aminobutyric acid; NSAIDs, nonsteroid anti-inflammatory drugs

${ }^{a}$ Data are presented as the number of prescriptions and number of patients with such prescriptions for different drug categories

relevant (which corresponds to the PPV) was comparable for the three CDSS, i.e., between 5.7 and $8 \%(p>0.1$ for all comparisons). The sensitivity to detect the 33 relevant interactions was $87.9 \%$ for DrugReax, $75.8 \%$ for TheraOpt, and 9.1\% for Pharmavista $(p<0.001$ for TheraOpt or DrugReax vs. Pharmavista; $p>0.1$ for DrugReax vs. TheraOpt). Further stratification by severity classification (Table 3 ) showed (1) a poor correlation between CDSS severity class and our evaluation of clinical significance for individual patients and (2) that DrugReax assigned a higher proportion of its alerts to a higher severity class than TheraOpt. A detailed description of all relevant interactions is presented in Table 4. Amiodarone was involved in eight interactions, antimycotics in seven, and statins and immunosuppressants in six each. Pharmacokinetic and pharmacodynamic interaction mechanisms were involved with an about equal frequency. For 19 of the 33 forwarded alerts (57.6\%), we observed a subsequent prescription change that was in line with our recommendations.

\section{Dosing}

TheraOpt is also able to identify dosing errors based on recommended maximum doses for specific indications, gender, age, and renal and liver function. We used this feature for an evaluation of the appropriate dosing for all prescriptions. TheraOpt recommended 31 dose adjustments, and we found one additional dosing problem related to a decreased first pass of metoprolol in cirrhosis. Among these 32 "dosing problems," we considered 11 as justified and forwarded recommendations for prescription changes, which were followed by changes in three cases (Table 5 ).

\section{Adverse drug events}

In four patients we detected adverse events that were possibly related to the identified interaction or dosing issues. In the first case, oral ciprofloxacin was combined with oral calcium, and the patient developed cholecystitis and Escherichia coli sepsis. Impaired absorption of ciprofloxacin may have contributed to treatment failure, leading to prolonged hospitalization in this case. In the second case, a patient developed hypokalemia $(2.9 \mathrm{mmol} / \mathrm{l})$ under combined therapy with hydrochlorothiazide, torasemide, and prednisone. After medication change, the patient's serum potassium quickly normalized. The third patient concomitantly received itraconazole capsules and pantoprazole. Proton pump inhibitors are known to impair the bioavailability of itraconazole capsules [26], and drug monitoring indeed showed subtherapeutic itraconazole concentrations $(0.2 \mathrm{mg} / 1$; target level is $>1 \mathrm{mg} / \mathrm{l}$ ). The fourth patient had a transjugular intrahepatic portosystemic shunt (TIPS) and received $6 \mathrm{mg}$ budesonide per day for autoimmune hepatitis with cirrhosis stage Child B. This patient developed a thrombosis of the TIPS. Elevated bioavailability of budesonide due to 
Fig. 2 Polypharmacy.

Histogram of the polypharmacy

distribution for 100 patients

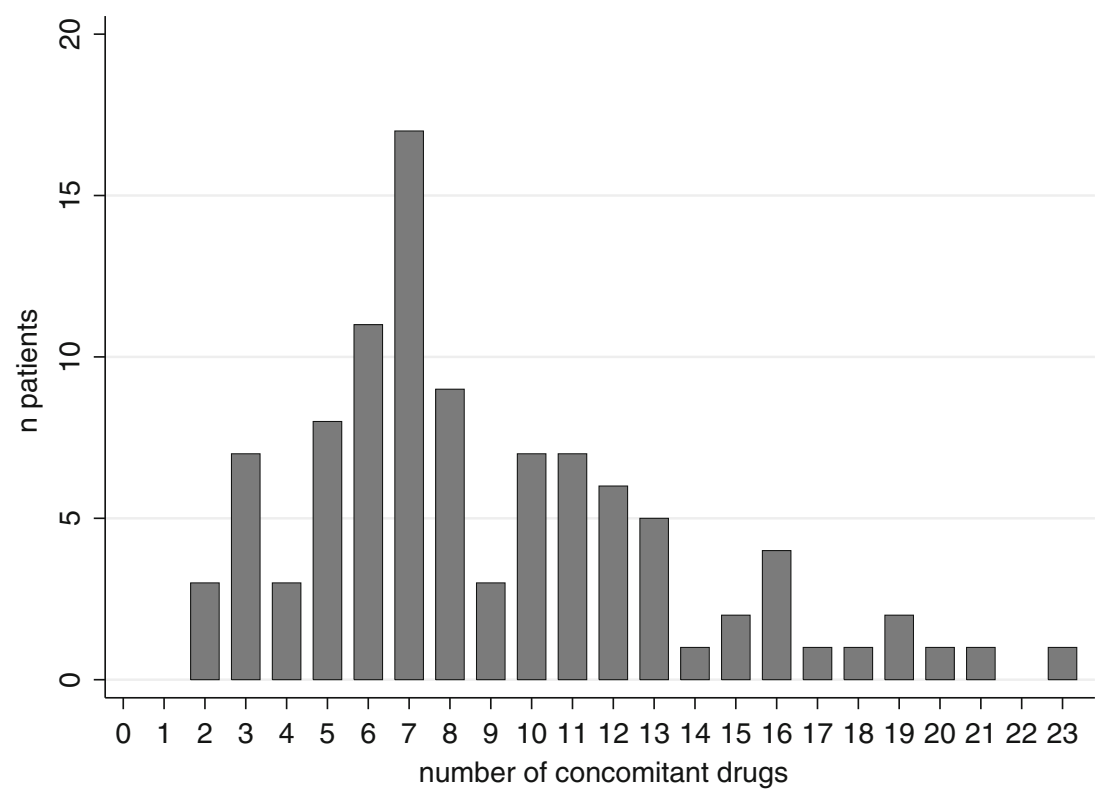

portocaval shunting in association with portal vein thrombosis has been described [27], and elevated concentrations may have contributed to TIPS thrombosis here.

\section{Discussion}

This study presents a systematic analysis of our experience with three different CDSS used as screening tools for medication errors in daily clinical routine.

First, our results provide information on the occurrence of specific medication errors in the studied setting. Amiodarone, antimycotics, cholesterol-lowering 3-hydroxy-3methylglutaryl-coenzyme A (HMG-CoA)-reductase inhibitors, and immunosuppressants were most frequently involved in relevant interactions, underlining that any prescribing physician should give particular attention to their interaction profiles and routinely check for interactions when prescribing these drugs. Of interest, a previous study using a different CDSS in a different population of 84,607 psychiatric inpatients also found amiodarone to have the highest intrinsic risk of interactions [28]. We also found some cases in which there was a need for dose adjustments. Most were related to renal insufficiency and some also to cirrhosis and high age, but there was no single drug frequently prescribed with an unadjusted dose. Overall, a total of 22 medication changes following our combined 44 recommendations relating to interactions or dosing in 100 patients suggest that pharmacotherapy could be improved in a considerable proportion and absolute number of hospitalized patients. Although our analysis was not designed and powered to demonstrate a reduction in clinical outcomes and costs, the detection of possible ADE in $4 \%$ of
Fig. 3 Polypharmacy and identification of drug interactions by CDSS. Mean number of identified interactions per patient for each CDSS across polypharmacy categories in 100 patients. Pharmavista did not allow analysis of more than eight concomitant drugs. n.a. Data not available

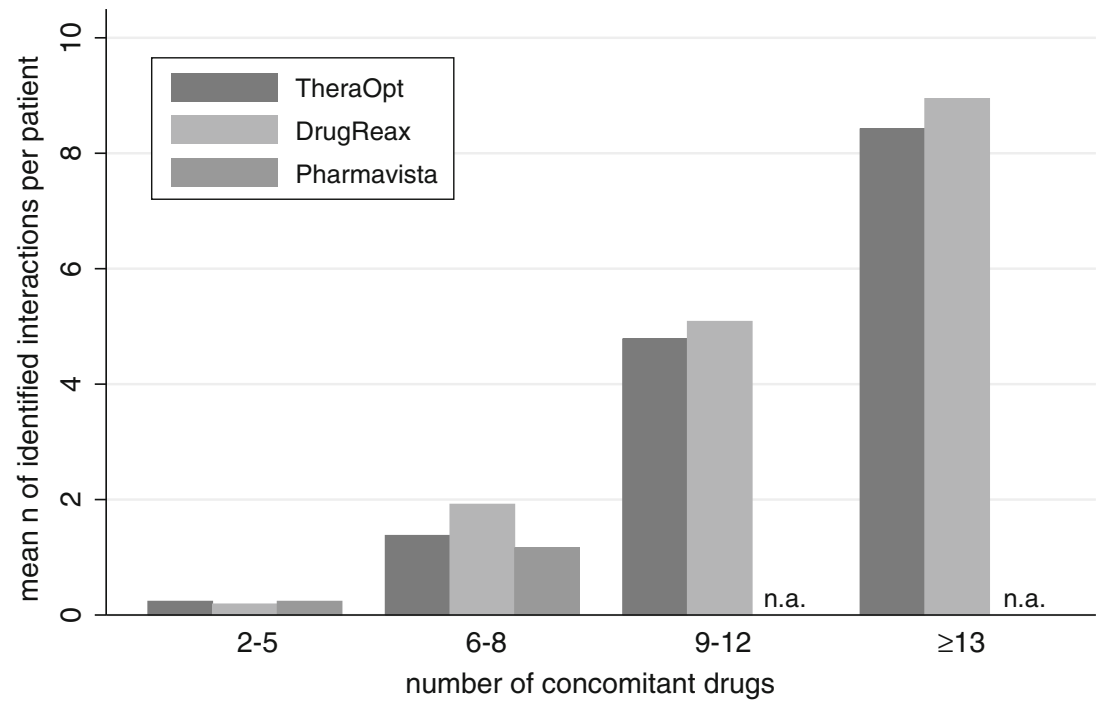


Table 3 Identification and evaluation of drug interactions ${ }^{\mathrm{a}}$

\begin{tabular}{|c|c|c|c|c|c|c|}
\hline \multirow[t]{2}{*}{ Identification and evaluation of interactions } & \multicolumn{2}{|c|}{ PharmaVista $^{\mathrm{b}}$} & \multicolumn{2}{|c|}{ DrugReax } & \multicolumn{2}{|c|}{ TheraOpt } \\
\hline & $n$ & $\%$ & $n$ & $\%$ & $n$ & $\%$ \\
\hline All interactions & 53 & 100 & 362 & 100 & 328 & 100 \\
\hline Evaluated as clinically relevant $\left(\mathrm{PPV}^{\mathrm{c}}\right)$ & 3 & 5.7 & 29 & 8.0 & 25 & 7.6 \\
\hline Sensitivity $^{\mathrm{d}}$ & & 9.1 & & 87.9 & & 75.8 \\
\hline Alert followed by prescription change & 3 & 5.7 & 17 & 4.7 & 13 & 4.0 \\
\hline Adverse event associated with interaction & 0 & 0 & 3 & 0.8 & 3 & 0.9 \\
\hline Mild interactions & 36 & 100 & 23 & 100 & 213 & 100 \\
\hline Evaluated as clinically relevant $\left(\mathrm{PPV}^{\mathrm{c}}\right)$ & 3 & 8.3 & 0 & 0 & 4 & 1.9 \\
\hline Sensitivity ${ }^{\mathrm{d}}$ & & 9.1 & & 0 & & 12.1 \\
\hline Alert followed by prescription change & 3 & 8.3 & - & - & 4 & 1.9 \\
\hline Adverse event associated with interaction & 0 & 0 & - & - & 0 & 0 \\
\hline Moderate interactions & 17 & 100 & 248 & 100 & 104 & 100 \\
\hline Evaluated as clinically relevant $\left(\mathrm{PPV}^{\mathrm{c}}\right)$ & 0 & 0 & 12 & 4.8 & 15 & 14.4 \\
\hline Sensitivity $^{\mathrm{d}}$ & & 0 & & 36.4 & & 42.4 \\
\hline Alert followed by prescription change & - & - & 5 & 2.0 & 5 & 4.8 \\
\hline Adverse event associated with interaction & - & - & 3 & 1.2 & 3 & 2.9 \\
\hline Severe interactions & 0 & - & 91 & 100 & 11 & 100 \\
\hline Evaluated as clinically relevant $\left(\mathrm{PPV}^{\mathrm{c}}\right)$ & - & - & 17 & 18.7 & 6 & 54.5 \\
\hline Sensitivity $^{\mathrm{d}}$ & & 0 & & 51.5 & & 18.2 \\
\hline Alert followed by prescription change & - & - & 12 & 13.2 & 4 & 36.4 \\
\hline Adverse event associated with interaction & - & - & 0 & 0 & 0 & 0 \\
\hline
\end{tabular}

${ }^{a}$ Identification of drug interactions by CDSS and further expert evaluation according prescription changes and associated adverse events. Overall results are presented first, followed by stratifications over the severity grading of interactions

${ }^{\mathrm{b}}$ Pharmavista did not analyze pharmacotherapy if the number of concomitant drugs was $>8$

${ }^{\mathrm{c}}$ Percentage value equals positive predictive value (PPV): PPV $=$ (number of interactions identified by the respective CDSS and evaluated as clinically relevant/number of interactions identified by the respective CDSS $) \times 100$

${ }^{\mathrm{d}}$ Sensitivity $(\%)=$ (number of interactions among all 33 interactions evaluated as clinically relevant identified by the respective CDSS/all 33 interactions identified by any source and evaluated as clinically relevant) $\times 100$

the population is in accordance with earlier reports [1-4]. Although ADE are relatively rare, an extrapolation of our results to the whole patient population of a hospital would yield a considerable absolute number of preventable ADE and therefore supports the view that local efforts to introduce preventive countermeasures should be increased.

Considering CDSS as such potentially appropriate countermeasures, the results of our study provide important insight into several areas of interest, i.e., the comparative performance, the applicability, and the clinical relevance and potential benefits of CDSS use in routine clinical practice. In contrast to the primarily intended use of CDSS by prescribers and office pharmacists, a key feature of our study is that we, as clinical pharmacologists, used CDSS as a screening tool for medication errors, whereas the prescribing physicians were not directly confronted with CDSS-generated alerts. Instead, we made a preselection of presumably clinically relevant alerts, based on both patient-specific information and our clinical expertise, and subsequently were able to enhance the automated alerts by additional management recommendations. The rationale for this approach is our own experience as well as an increasing number of reports in the literature [22, 29-34] that have identified overalerting as a major issue with currently available CDSS. Indeed, although most alerts could be considered as generally valid, for all three CDSS that we used more than $90 \%$ of alerts appeared to be clinically irrelevant when they were applied to the management of specific patients. Therefore, also in our setting, forwarding all alerts without selection would have most likely led to indiscriminate overriding. Furthermore, our finding that many clinically relevant interactions were classified as moderate or even mild according to the classic "traffic light" grading used by all three CDSS indicates that even filtering only "severe" alerts would not be a reasonable solution. Indeed, a previous study also reported that customization of CDSS by common severity levels was not able to improve effectiveness [32]. The more management-oriented Operational 
Table 4 Listing of all drug interactions that were identified by CDSS and evaluated as clinically relevant during expert discussion ${ }^{\mathrm{a}}$

\begin{tabular}{|c|c|c|c|c|c|c|}
\hline \multirow[t]{2}{*}{ Interacting drugs } & \multicolumn{3}{|c|}{$\begin{array}{l}\text { CDSS detection and } \\
\text { severity grading }\end{array}$} & \multirow[t]{2}{*}{ Potential adverse event } & \multirow[t]{2}{*}{ Mechanism $^{c}$} & \multirow[t]{2}{*}{ Prescription change } \\
\hline & $\mathrm{PhVis}$ & DRx & ThOpt & & & \\
\hline Amiodarone-simvastatin & $\mathrm{x}$ & 3 & 2 & Myopathy & PK & No \\
\hline Amiodarone-atorvastatin & $\mathrm{x}$ & 2 & 2 & Myopathy & PK & Yes \\
\hline Amiodarone-metronidazole & $\mathrm{x}$ & 3 & $\mathrm{x}$ & QT-prolongation & $\mathrm{PD}$ & Yes \\
\hline Amiodarone-cotrimoxazole & $\mathrm{x}$ & 3 & 3 & QT-prolongation & PD & Yes \\
\hline Amiodarone-itraconazole & $\mathrm{x}$ & 3 & $\mathrm{x}$ & QT-prolongation & $\mathrm{PD}$ & Yes \\
\hline Amiodarone-clarithromycin $(n=2)$ & $\mathrm{x}$ & 3 & 3 & QT-prolongation & PD & $2 \times$ yes \\
\hline Amiodarone-ciprofloxacin & $\mathrm{x}$ & 3 & $\mathrm{x}$ & QT-prolongation & PD & Yes \\
\hline Cyclosporine-simvastatin & $\mathrm{x}$ & 3 & 2 & Myopathy & PK & Yes \\
\hline Cyclosporine-pravastatin & $\mathrm{x}$ & 2 & 2 & Myopathy & PK & No \\
\hline Cyclosporine-morphine-loperamide & $\mathrm{x}$ & $\mathrm{x}$ & $\mathrm{x}$ & $\begin{array}{l}\text { Increased levels of cyclosporine } \\
\text { and morphine }\end{array}$ & PK & No \\
\hline Tacrolimus-itraconazole & $\mathrm{x}$ & 3 & 2 & Increased levels of tacrolimus & PK & No \\
\hline Tacrolimus-clarithromycin & $\mathrm{x}$ & 2 & 2 & Increased levels of both drugs & PK & No \\
\hline Tacrolimus-metronidazol & $\mathrm{x}$ & 2 & $\mathrm{x}$ & Increased levels of tacrolimus & PK & No \\
\hline Clarithromycin-domperidone-itraconazol & $\mathrm{x}$ & $\mathrm{x}$ & 2 & QT-prolongation & PK & Yes \\
\hline Clarithromycin-cotrimoxazole $(n=3)$ & $\mathrm{x}$ & 3 & 3 & QT-prolongation & PD & $1 \times$ yes, $2 \times$ no \\
\hline Cotrimoxazole-fluconazole & $\mathrm{x}$ & 3 & $\mathrm{x}$ & QT-prolongation & PD & 1 \\
\hline Ciprofloxacin-atorvastatin & $\mathrm{x}$ & 2 & 2 & Myopathy & PK & No \\
\hline Ciprofloxacin-calcium $^{\mathrm{d}}(n=2)$ & $\mathrm{x}$ & 2 & 2 & Decreased efficacy $\left(B V^{e} \downarrow\right)$ & PK & Yes/no \\
\hline Itraconazole-ranitidine & $\mathrm{x}$ & 2 & 2 & Decreased efficacy $\left(B V^{e} \downarrow\right)$ & PK & No \\
\hline Itraconazol-omeprazole & $\mathrm{x}$ & 2 & 2 & Decreased efficacy $\left(B V^{e} \downarrow\right)$ & PK & No \\
\hline Itraconazole-pantoprazole ${ }^{\mathrm{d}}$ & $\mathrm{x}$ & 2 & 2 & Decreased efficacy $\left(B V^{e} \downarrow\right)$ & PK & No \\
\hline Pipamperone-trimipramine & $\mathrm{x}$ & 3 & 2 & QT-prolongation & PD & No \\
\hline Ursodeoxycholic acid-colestyramin & 1 & $\mathrm{x}$ & 1 & Decreased efficacy $\left(B V^{e} \downarrow\right)$ & PK & Yes \\
\hline Daptomycin-pravastatin & $\mathrm{x}$ & $\mathrm{x}$ & 2 & Myopathy & PD & Yes \\
\hline Torasemide-HCTZ-prednisone ${ }^{\mathrm{d}}$ & $\mathrm{x}$ & 2 & 1 & Hypokalemia & PD & Yes \\
\hline Ginkgo biloba-acetylsalicylic acid & 1 & 3 & 1 & Bleeding & PD & Yes \\
\hline Ginkgo biloba-clopidogrel & 1 & 2 & 1 & Bleeding & PD & Yes \\
\hline Clopidogrel-esomeprazole & $\mathrm{x}$ & 3 & $\mathrm{x}$ & Decreased clopidogrel efficacy & PK & Yes \\
\hline Terlipressin-quetiapine & $\mathrm{x}$ & 3 & $\mathrm{x}$ & QT-prolongation & PD & Yes \\
\hline
\end{tabular}

${ }^{\text {a }}$ Twenty-nine distinct interactions occurring in 33 instances

${ }^{\mathrm{b}}$ Key for severity grading: 1 = mild, 2 = moderate, 3 = severe, $\mathrm{x}$ = not detected; PhVis, Pharmavista; DRx, DrugReax; ThOpt, Theraopt

c PK, Pharmacokinetic; PD, pharmacodynamic

${ }^{\mathrm{d}}$ Associated with adverse event (see text)

e BV, Bioavailability, refers to the first drug of the listed combination

Classification of Drug Interactions (ORCA) has been developed with this issue in mind [35], and recently we proposed an extension of ORCA that also supports the implementation of patient-specific information and potential outcomes of drug interactions in CDSS algorithms [28, 36, 37]. Currently ongoing studies that evaluate CDSS based on such management-oriented classifications will show whether they can make a relevant contribution to the reduction of overalerting.
A comparison of the three CDSS in terms of their sensitivity to detect clinically relevant interactions shows that DrugReax and TheraOpt performed better than Pharmavista. Interpretation of this result must certainly take into account Pharmavista's inability to analyze more than eight drugs concomitantly, which applied to $42 \%$ of our medical in-patient population, and it may be less of an issue in a pharmacy setting, where Pharmavista is frequently used. Nevertheless, our results indicate that 
Table 5 Dosing recommendations initially identified by TheraOpt and subsequently evaluated as clinically relevant

GFR, Glomerular filtration rate ${ }^{\mathrm{a}} n=1$ for all dosing errors with the exception of zolpidem $10 \mathrm{mg}$ $(n=2)$

${ }^{\mathrm{b}}$ Associated with adverse event (see text)

\begin{tabular}{lll}
\hline Actual prescription $^{\mathrm{a}}$ & Dosing issue & Prescription change \\
\hline Zolpidem $10 \mathrm{mg}$ & $>65$ years standard dose $5 \mathrm{mg}$ & Yes/no \\
Chlortalidon & Contraindicated in severe renal impairment & Yes \\
Budesonide $^{\mathrm{b}}$ & In cirrhosis high first pass & Yes \\
Hydrochlorothiazide & Contraindicated in severe renal impairment & No \\
Metformin & Contraindicated in severe renal impairment & No \\
Simvastatin $40 \mathrm{mg}$ & Maximum 10 mg if GFR $<30$ & No \\
Metamizole $3 \mathrm{~g}$ & Lower dose in renal impairment & No \\
Pantoprazole $40 \mathrm{mg}$ & In cirrhosis, maximum $20 \mathrm{mg}$ & No \\
Clarithromycin $2 \times 250 \mathrm{mg}$ & Maximum $250 \mathrm{mg}$ if GFR $<30$ & No \\
\hline
\end{tabular}

it is quite a relevant limitation for a medical in-patient population.

Among all of the interaction alerts that we considered as relevant, 58\% ultimately resulted in a prescription change. This demonstrates that our preselection of alerts based on patient-specific clinical information and our proposals of management recommendations in combination with personal communication of the alerts were generally well received by the attending physicians and able to overcome the major problem of overriding drug safety alerts to a large part. For the remaining $42 \%$ of alerts that were not followed by a prescription change, clinicians may have either judged our recommendations as not justified (which may or may not be true), or indiscriminative overriding may have occurred. Previous studies have identified the most frequent reasons for drug safety alert overriding as: (1) the problem was already known but clinically not important; (2) there were no reasonable alternatives while the benefit was considered to be greater than the risk; (3) the potential problem could be managed by appropriate monitoring; (4) the dose had been adjusted; (5) the patient had previously tolerated the medication [31, 32]. Similar reasons were brought forward when we contacted the prescribers. We also realize that there are a number of unavoidable limitations to our assessments, such as the fact that in the absence of a gold standard for the clinical relevance of potential medication errors, it is possible that we excluded some relevant alerts, oversaw additional medication errors or, on the other hand, forwarded some irrelevant alerts. However, we neither identified additional ADE, nor does it appear likely that the combined sensitivity of the three CDSS plus our manual review failed to identify a relevant number of medication errors.

Regardless of the reason for overriding safety drug alerts and the differences that we found between CDSS, the fact that less than $10 \%$ of automated alerts were assessed as relevant for individual patients forces us to rethink how future CDSS can be effectively and efficiently applied for the prevention of medications errors, $\mathrm{ADE}$, and unnecessary costs in clinical practice. Maximum sensitivity may be a common priority in the development of CDSS, and much of the content of such systems can be tracked down to the safety information included in the manufacturer's prescribing information where, also for legal reasons, comprehensiveness rather than clinical relevance is determinative. The comprehensive and generally valid alerts and comments from all three CDSS were certainly useful for screening and learning purposes in our setting. However, eventually CDSS must be integrated with CPOE and automatically display alerts that have a high propensity of clinical relevance. Indeed, approaches that target specific relevant problems of pharmacotherapy have demonstrated their efficacy not only in modifying prescribing behavior but also in improving clinical outcomes [38]. Therefore, we propose that, on the one hand, the valuable comprehensive knowledge from CDSS should be easily available (via "one click") to the prescriber, but only on an on-demand basis. On the other hand, there should be a list with a limited number of automatically computer-triggered alerts. Clinical pharmacologists can initially develop such a list and make a preselection that focuses on clinical relevance and management implications. Local retrospective systematic evaluations of medication errors and ADE that occurred in the past can also contribute to and help to justify the use of such a list [36, 37]. However, the list should also be reviewed and supported by local leading clinicians in order to enhance its local acceptance. Furthermore, the importance of patientspecific factors in our study suggests that alert algorithms must also include as much laboratory and clinical information as possible. Indeed, obtaining laboratory results as part of intensified monitoring could also be part of clinical actions recommended and surveyed by CDSS. This requires interfaces that integrate CDSS with laboratory and clinical information systems. For example, alert algorithms may include current electrolyte results and QTc times from automated electrocardiogram readings. Although we did not use CDSS with integration into CPOE and clinical information systems in our setting, it is important to note that TheraOpt and other currently available CDSS have indeed been 
designed in this a way. However, if this approach is used for all interactions of a highly sensitive CDSS, its complexity and applicability will likely become uncontrollable and result once more in overriding of the drug safety alerts.

\section{Conclusions}

The studied CDSS are valuable screening tools for medication errors, but only a small fraction of all alerts are clinically relevant in individual patients. Insufficient use of patient-specific information in alert algorithms and lack of local customization compromise their applicability and efficacy in clinical practice. In addition, lack of scientific evidence in complex individual patient care and common offlabel use remain major challenges for CDSS. We therefore propose that CDSS should separate comprehensive ondemand information from selected computer-triggered alerts that must be locally supported, customized and frequently updated, and have management implications in the majority of patients where they are displayed. The initial setup plus necessary ongoing evaluations and adjustments of such a system requires expertise and additional resources. However, compared to a standard CDSS without appropriate local promotion and support, such an approach may not only be more efficacious but also more efficient.

Acknowledgments The physicians at the Department of Internal Medicine at the University Hospital Zurich are gratefully acknowledged for their time and effort spent in discussing and improving the patients' pharmacotherapy.

Financial support and conflict of interest statement The work presented in this manuscript was carried out independently by the authors and with general resources available at the Department of Clinical Pharmacology. IC, ME, and GKU are involved in the development of prescribing software, but they have no financial associations to the CDSS studied here. All authors declare that they have no conflict of interest regarding the work presented here.

\section{References}

1. Bates DW, Cullen DJ, Laird N, Petersen LA, Small SD, Servi D, Laffel G, Sweitzer BJ, Shea BF, Hallisey R et al (1995) Incidence of adverse drug events and potential adverse drug events. implications for prevention. ADE Prevention Study Group. JAMA 274(1):29-34

2. Classen DC, Pestotnik SL, Evans RS, Lloyd JF, Burke JP (1997) Adverse drug events in hospitalized patients. excess length of stay, extra costs, and attributable mortality. JAMA 277(4):301-306

3. Lagnaoui R, Moore N, Fach J, Longy-Boursier M, Begaud B (2000) Adverse drug reactions in a department of systemic diseases-oriented internal medicine: prevalence, incidence, direct costs and avoidability. Eur J Clin Pharmacol 56(2):181-186

4. Lazarou J, Pomeranz BH, Corey PN (1998) Incidence of adverse drug reactions in hospitalized patients: a meta-analysis of prospective studies. JAMA 279(15):1200-1205
5. Pirmohamed M, James S, Meakin S, Green C, Scott AK, Walley TJ, Farrar K, Park BK, Breckenridge AM (2004) Adverse drug reactions as cause of admission to hospital: prospective analysis of 18820 patients. Br Med J 329(7456):15-19

6. Juurlink DN, Mamdani M, Kopp A, Laupacis A, Redelmeier DA (2003) Drug-drug interactions among elderly patients hospitalized for drug toxicity. JAMA 289(13):1652-1658

7. Juurlink DN, Mamdani MM, Lee DS, Kopp A, Austin PC, Laupacis A, Redelmeier DA (2004) Rates of hyperkalemia after publication of the randomized aldactone evaluation study. N Engl J Med 351 (6):543-551

8. Ammenwerth E, Schnell-Inderst P, Machan C, Siebert U (2008) The effect of electronic prescribing on medication errors and adverse drug events: a systematic review. J Am Med Inform Assoc 15(5):585-600

9. Feldstein AC, Smith DH, Perrin N, Yang X, Rix M, Raebel MA, Magid DJ, Simon SR, Soumerai SB (2006) Improved therapeutic monitoring with several interventions: a randomized trial. Arch Intern Med 166(17):1848-1854

10. Kaushal R, Kern LM, Barron Y, Quaresimo J, Abramson EL (2010) Electronic prescribing improves medication safety in community-based office practices. J Gen Intern Med 25(6):530 536

11. Kaushal R, Shojania KG, Bates DW (2003) Effects of computerized physician order entry and clinical decision support systems on medication safety: a systematic review. Arch Intern Med 163(12):1409-1416

12. Smith DH, Perrin N, Feldstein A, Yang X, Kuang D, Simon SR, Sittig DF, Platt R, Soumerai SB (2006) The impact of prescribing safety alerts for elderly persons in an electronic medical record: an interrupted time series evaluation. Arch Intern Med 166(10):1098-1104

13. Eslami S, de Keizer NF, Abu-Hanna A (2008) The impact of computerized physician medication order entry in hospitalized patients - a systematic review. Int J Med Inform 77(6):365-376

14. van Doormaal JE, van den Bemt PM, Zaal RJ, Egberts AC, Lenderink BW, Kosterink JG, Haaijer-Ruskamp FM, Mol PG (2009) The influence that electronic prescribing has on medication errors and preventable adverse drug events: an interrupted timeseries study. J Am Med Inform Assoc 16(6):816-825

15. Wolfstadt JI, Gurwitz JH, Field TS, Lee M, Kalkar S, Wu W, Rochon PA (2008) The effect of computerized physician order entry with clinical decision support on the rates of adverse drug events: a systematic review. J Gen Intern Med 23(4):451-458

16. Olvey EL, Clauschee S, Malone DC (2010) Comparison of critical drug-drug interaction listings: the Department of Veterans Affairs medical system and standard reference compendia. Clin Pharmacol Ther 87(1):48-51

17. Abarca J, Malone DC, Armstrong EP, Grizzle AJ, Hansten PD, Van Bergen RC, Lipton RB (2004) Concordance of severity ratings provided in four drug interaction compendia. J Am Pharm Assoc 44(2):136-141

18. Vonbach P, Dubied A, Krahenbuhl S, Beer JH (2008) Evaluation of frequently used drug interaction screening programs. Pharm World Sci 30(4):367-374

19. van Doormaal JE, Mol PG, van den Bemt PM, Zaal RJ, Egberts AC, Kosterink JG, Haaijer-Ruskamp FM (2008) Reliability of the assessment of preventable adverse drug events in daily clinical practice. Pharmacoepidemiol Drug Saf 17(7):645-654

20. Smithburger PL, Kane-Gill SL, Benedict NJ, Falcione BA, Seybert AL (2010) Grading the severity of drug-drug interactions in the intensive care unit: a comparison between clinician assessment and proprietary database severity rankings. Ann Pharmacother 44 (11):1718-1724

21. Vonbach P, Dubied A, Beer JH, Krahenbuhl S (2007) Recognition and management of potential drug-drug interactions in patients on internal medicine wards. Eur J Clin Pharmacol 63(11):1075-1083 
22. van der Sijs H, Mulder A, van Gelder T, Aarts J, Berg M, Vulto A (2009) Drug safety alert generation and overriding in a large Dutch University Medical Centre. Pharmacoepidemiol Drug Saf 18 (10):941-947

23. Thomson Reuters DRUG-REAX SYSTEM. Available at: http:// thomsonreuters.com/products_services/healthcare/healthcare products/a-z/drug_reax_system

24. Pharmavista Interactions (2011). Available at: http://www. pharmavista.ch

25. ID Berlin ID PHARMA CHECK. Available at: http://www. id-berlin.de

26. Albengres E, Le Louet H, Tillement JP (1998) Systemic antifungal agents. drug interactions of clinical significance. Drug Saf 18(2):83-97

27. Hempfling W, Grunhage F, Dilger K, Reichel C, Beuers U, Sauerbruch T (2003) Pharmacokinetics and pharmacodynamic action of budesonide in early- and late-stage primary biliary cirrhosis. Hepatology 38 (1):196-202

28. Haueis P, Greil W, Huber M, Grohmann R, Kullak-Ublick GA, Russmann S (2011) Evaluation of drug interactions in a large sample of psychiatric inpatients: a data interface for mass analysis with clinical decision support software. Clin Pharmacol Ther 90 (4):588-596

29. Taylor LK, Tamblyn R (2004) Reasons for physician nonadherence to electronic drug alerts. Stud Health Technol Inform 107(Pt 2):1101-1105

30. Weingart SN, Toth M, Sands DZ, Aronson MD, Davis RB, Phillips RS (2003) Physicians' decisions to override computerized drug alerts in primary care. Arch Intern Med 163(21):2625-2631

31. Shah NR, Seger AC, Seger DL, Fiskio JM, Kuperman GJ, Blumenfeld B, Recklet EG, Bates DW, Gandhi TK (2006) Improving acceptance of computerized prescribing alerts in ambulatory care. J Am Med Inform Assoc 13(1):5-11
32. Tamblyn R, Huang A, Taylor L, Kawasumi Y, Bartlett G, Grad R, Jacques A, Dawes M, Abrahamowicz M, Perreault R, Winslade N, Poissant L, Pinsonneault A (2008) A randomized trial of the effectiveness of on-demand versus computer-triggered drug decision support in primary care. J Am Med Inform Assoc 15(4):430 438

33. Isaac T, Weissman JS, Davis RB, Massagli M, Cyrulik A, Sands DZ, Weingart SN (2009) Overrides of medication alerts in ambulatory care. Arch Intern Med 169(3):305-311

34. Weingart SN, Simchowitz B, Shiman L, Brouillard D, Cyrulik A, Davis RB, Isaac T, Massagli M, Morway L, Sands DZ, Spencer J, Weissman JS (2009) Clinicians' assessments of electronic medication safety alerts in ambulatory care. Arch Intern Med 169(17):16271632

35. Hansten PD, Horn JR, Hazlet TK (2001) ORCA: OpeRational ClassificAtion of drug interactions. J Am Pharm Assoc (Wash) 41 (2):161-165

36. Frölich T, Zorina O, Fontana AO, Kullak-Ublick GA, Vollenweider A, Russmann S (2011) Evaluation of medication safety in the discharge medication of 509 surgical inpatients using electronic prescription support software and an extended operational interaction classification. Eur J Clin Pharmacol 67:1273-1282

37. Guzek M, Zorina OI, Semmler A, Gonzenbach RR, Huber M, Kullak-Ublick GA, Weller M, Russmann S (2011) Evaluation of drug interactions and dosing in 484 neurological inpatients using clinical decision support software and an extended operational interaction classification system (Zurich Interaction System). Pharmacoepidemiol Drug Saf 20(9):930-938

38. Kucher N, Koo S, Quiroz R, Cooper JM, Paterno MD, Soukonnikov B, Goldhaber SZ (2005) Electronic alerts to prevent venous thromboembolism among hospitalized patients. N Engl J Med 352 (10):969-977 